\section{Attenuation of Cerebral Veins in Susceptibility-Weighted MR Imaging Performed with the Patient under General Anesthesia}

One of the most important advantages of susceptibility-weighted MR Imaging (SWI) is the exquisite demonstration of cerebral venous anatomy. ${ }^{1,2}$ This is useful for detecting developmental venous anomalies and in conditions associated with venous anomalies such as Sturge-Weber syndrome. The demonstration of accentuation of veins due to venous stasis in venous thrombosis or increased oxygen extraction in focal infraction has been described. ${ }^{1}$

In our pediatric patients in whom MR imaging is performed with the patient under general anesthesia, we have noted that the SWI shows the venous anatomy poorly (Fig $1 A,-B$ ) in comparison with those patients in whom the study was performed without anesthesia (Fig 1C). Anesthesia is induced in most of these children with $2 \%-3 \%$ sevoflurane in oxygen, by using a modified Bains circuit and titrated to the loss of eyelash reflex. In the MR imaging suite, anesthesia is continued with sevoflurane in a 1:1 mixture of air-oxygen, administered through the auxiliary gas outlet of the MR imaging-compatible Datex-Ohmeda Aestiva/5 MRI anesthesia ventilator (GE Healthcare, Madison, Wis), with the children breathing spontaneously. In a few of these patients, anesthesia was induced by using intramuscular injection of ketamine and midazolam.

The contrast mechanism in SWI is primarily associated with the magnetic-susceptibility difference between oxygenated and deoxygenated hemoglobin, leading to a phase difference between regions containing deoxygenated blood and surrounding tissues, resulting in signal-intensity cancellation. During anesthesia, on the basis of the depth of anesthesia, the oxygen extraction in the brain tissue is decreased. This decrease, in turn, results in a change in the extent and amplitude of the blood oxygen level-dependent response as has been demonstrated in earlier functional MR imaging studies. ${ }^{3}$

Initially, we believed that a decrease in deoxyhemoglobin within cerebral veins secondary to oxygen inhalation during anesthesia was responsible for the attenuation of signal intensity. If this hypothesis is correct, then oxygen inhalation can be used for differentiating hemorrhage from vein in SWI. Earlier studies have shown that SWI performed before and after administration of intravenous gadolinium can distinguish both. ${ }^{4}$ To test this hypothesis, we performed SWI of the brain in an adult patient without and with oxygen inhalation through a mask, which decreased the venous signals, but only marginally.

Retrospectively when we reviewed the SWIs of patients under anesthesia, we noted that the attenuation of signal intensity was present in all the patients but varied among patients. This made us think that the depth of anesthesia is probably responsible for this variation. Therefore, we think that cerebral oxygen extraction depending on cerebral metabolism and, to a lesser extent, oxygen saturation contribute to the observed SWI signal intensity. We are planning prospective studies on the influence of the depth of anesthesia on signalintensity attenuation of veins in SWI.

\section{References}

1. Tong KA, Ashwal S, Obenaus A, et al. Susceptibility-weighted MR imaging: a review of clinical applications in children. AJNR Am J Neuroradiol 2008;29:9-17

2. Thomas B, Somasundaram S, Thamburaj K, et al. Clinical applications of susceptibility weighted MR imaging of the brain: a pictorial review. Neuroradiology 2008;50:105-16. Epub 2007 Oct 11.

3. Marcar VL, Schwarz U, Martin E, et al. How depth of anesthesia influences the blood oxygenation level-dependent signal from the visual cortex of children. AJNR Am J Neuroradiol 2006;27:799-805

4. Lin W, Mukherjee P, An H, et al. Improving high-resolution MR bold venographic imaging using a T1 reducing contrast agent. J Magn Reson Imaging 1999; 10:118-23

C. Kesavadas

B. Thomas

S. Misra

J. Saini

Sree Chitra Tirunal Institute for Medical Sciences and Technology

Trivandrum, India

D0I 10.3174/ajnr.A1083
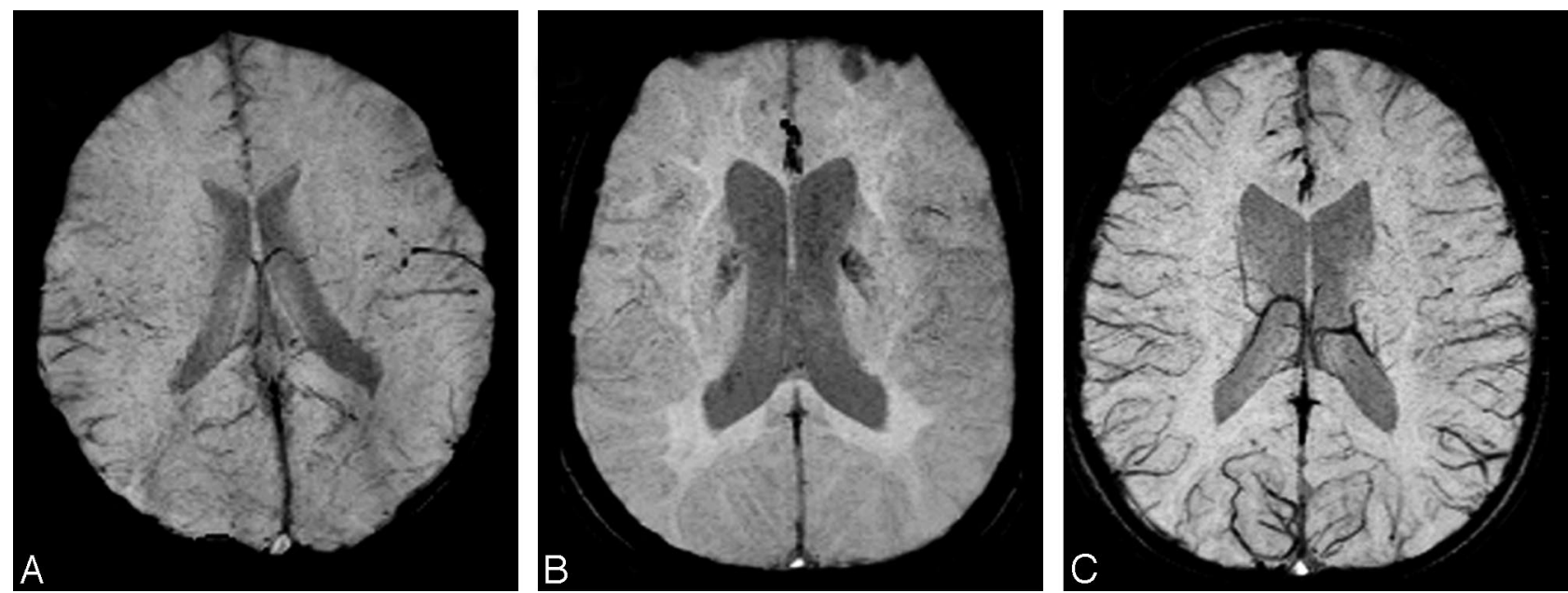

Fig 1. SWI minimal-intensity projections. A, A 6-year-old child in whom SWI was performed after anesthesia using sevoflurane. B, A 17-year-old boy in whom SWI was performed after anesthesia using ketamine and midazolam. C, A 6-year-old boy in whom SWI was performed without anesthesia. 